\title{
Formação e estabilização de agregados pelo sistema radicular de braquiária em um Nitossolo Vermelho
}

\author{
Formation and stabilization of aggregates by the grass root system in an Oxisol
}

\author{
Eliane Duarte Brandão ${ }^{\mathrm{I}}$ Ivandro de França da Silva ${ }^{\mathrm{II}}$
}

RESUMO

As gramíneas atuam de maneira direta na formação e na estabilização de agregados do solo, devido à maior densidade de raízes e à liberação de exsudatos orgânicos no solo. O objetivo do trabalho foi avaliar os efeitos do sistema radicular da Brachiaria ruziziensis Germain et Evrard na formação e na estabilização de agregados de um Nitossolo Vermelho. O experimento foi conduzido em abrigo telado, utilizando-se solo coletado na camada de $0-20 \mathrm{~cm}$ de profundidade. $O$ solo foi destorroado manualmente e passado em peneira de $2,00 \mathrm{~mm}$ de abertura de malha e, posteriormente, separadas por classes de diâmetro de agregados de 2,00-1,00, 1,00-0,50 e 0,50-0,25 mm com auxílio de peneiras de malhas específicas. $O$ delineamento utilizado foi o inteiramente casualizado, em esquema fatorial $3 \times 2 \times 2 \times 4$, correspondendo a três classes de diâmetro de agregados (2,00-1,00, 1,00-0,50 e 0,50-0,25mm), duas condições de cultivo (com e sem gramínea), dois conteúdos de umidade (100\% e 60\% de água disponível) e quatro épocas de avaliação (90, 180, 270 e 360 dias após o plantio da gramínea), com quatro repetições. As amostras de agregados foram acondicionadas em vasos de polietileno com capacidade para $10 \mathrm{~kg}$. O conteúdo de umidade foi equilibrado através de irrigações a cada dois dias. As amostragens foram realizadas a cada três meses, determinandose o diâmetro médio ponderado de agregados secos ao ar (DMPAs), o diâmetro médio ponderado de agregado estáveis em água (DMPAu) e o índice de estabilidade dos agregados $(I E A=D M P A u / D M P A s)$. Verificaram-se maiores valores de DMPAu e de IEA para o tratamento com gramínea, indicando que o sistema radicular da Brachiaria ruziziensis favoreceu maior formação e estabilização dos agregados no solo.

Palavras-chave: Brachiaria ruziziensis, raízes, agregação do solo, água disponível.

\begin{abstract}
Grasses take direct action in the formation and stabilization of soil aggregates due to the density of roots and the release of organic exudates in soil. The objective of this study was to evaluate the effects of the root system of Brachiaria ruziziensis Germain et Evrard in the formation and stabilization of aggregates of an Oxisol. The experiment was conducted under greenhouse, using soil collected in the upper $0-20 \mathrm{~cm}$ depth. Loosening the soil was manually and passed through a sieve of $2.00 \mathrm{~mm}$ mesh size and subsequently separated by diameter classes of aggregates from 2.00-1.00, 1.00-0.50 and $0.50-0.25 \mathrm{~mm}$, with the help of specific mesh sieves. The experimental design was completely randomized in factorial $3 \times 2 \times 2 \times 4$, corresponding to three diameter classes of aggregates (2.00-1.00, $1.00-0.50$ and from $0.50-0.25 \mathrm{~mm})$, two conditions crop (with and without grass), two moisture contents (100\% and $60 \%$ of available water), and four sampling times (90, 180, 270 and 360 days after planting the grass), with four repetitions. The aggregate samples were packed in polyethylene vessels with a capacity of $10 \mathrm{~kg}$. The moisture content was balanced through irrigation every two days. Samples were taken every three months, determining the mean diameter of airdried aggregates (DMPAs), the mean weight diameter of stable aggregates in water (DMPAu) and aggregate stability index (IAS = DMPAu/DMPAs). It was found higher values of DMPAu and IAS to treatment with grass, indicating that the root system of Brachiaria ruziziensis favored increased formation and stabilization of soil aggregates.
\end{abstract}

Key words: Brachiaria ruziziensis, roots, soil aggregation, water available.

'Programa de Pós-graduação em Agronomia, Centro de Ciências Agrárias (CCA), Universidade Federal da Paraíba (UFPB), 58397000, Areia, PB, Brasil. E-mail: eliduartebrandao@yahoo.com.br. Autor para correspondência.

"Departamento de Solos e Engenharia Rural, CCA, UFPB, Areia, PB, Brasil. 


\section{INTRODUÇÃO}

A formação de agregados no solo ocorre, de maneira geral, em duas etapas: a primeira corresponde à aproximação das partículas do solo, decorrente das variações do conteúdo de água, favorecida pelos ciclos de umedecimento e secagem, pela ação mecânica das raízes e hifas de fungos ou pela atração eletrostática entre as partículas de solo (BOCHNER et al., 2008); a segunda se refere à estabilização, ou seja, à consolidação da união entre as partículas do solo pela ação cimentante dos polímeros orgânicos da matéria orgânica do solo e dos exsudados orgânicos liberados pelas raízes de plantas, em função das ligações com a superfície das partículas minerais do solo por meio de cátions polivalentes (CASTRO FILHO et al., 1998; MIELNICZUK, 1999).

Neste processo, seguindo a escala de hierarquização, primeiramente são formados os agregados $<0,25 \mathrm{~mm}$ (microagregados) pela repetida interação de moléculas orgânicas, cátions polivalentes e partículas minerais da fração argila (EDWARDS \& BREMNER, 1967), principalmente caulinita e óxidos de Fe, nos solos oxídicos (VEZZANI \& MIELNICZUK, 2011). Em seguida, os agregados $>0,25 \mathrm{~mm}$ (macroagregados) são formados pela união mecânica dos microagregados durante o crescimento das raízes de plantas vivas e hifas de fungos rizosféricos (TISDALL \& OADES, 1982; TISDALL, 1994).

As raízes de plantas aceleram a agregação, tanto pela liberação de exsudados orgânicos que servem de energia para a atividade microbiana, como pela sua atuação na aproximação das partículas minerais pelas pressões exercidas durante seu crescimento e na absorção de água, favorecendo a coesão entre as partículas do solo (CASTRO FILHO et al., 1998; SILVA et al., 1998; PALMEIRA et al., 1999). Pesquisas realizadas por SILVA\& MIELNICZUK (1997), CAMARGOFILHO (1999) e WOHLENBERG et al. (2004) demonstraram que as gramíneas contribuem nos processos de formação e estabilização de agregados do solo, devido, principalmente, à alta densidade de suas raízes, que promove a aproximação de partículas pela constante absorção de água do solo, como também às periódicas renovações do sistema radicular e à uniforme distribuição dos exsudados orgânicos ao solo, que estimulam a atividade microbiana, cujos subprodutos também atuam na estabilização dos agregados.

Como normalmente é analisado o efeito das gramíneas em toda a massa de solo, não sendo possível estimar sobre as diferentes classes de agregados, foi conduzido um trabalho com o objetivo de avaliar o comportamento do sistema radicular da Brachiaria ruziziensis Germain et Evrard na formação e na estabilização de agregados de diferentes classes de diâmetro, em um Nitossolo Vermelho.

\section{MATERIAL E MÉTODOS}

O experimento foi conduzido durante o período de um ano em um abrigo telado na Estação Experimental Chã de Jardim, do Centro de Ciências Agrárias da Universidade Federal da Paraíba, situado no município de Areia (latitude $06^{\circ} 58^{\prime}$ S , longitude $35^{\circ} 42^{\prime}$ $\mathrm{W}$ e altitude variando entre $400 \mathrm{~m}$ e $600 \mathrm{~m}$ ). O solo utilizado no experimento foi coletado no município de Alagoinha-PB, na camada de $0-20 \mathrm{~cm}$ de profundidade, com auxílio de enxadeco e pá de corte, em uma área com pastagem degradada, constituída predominantemente por capim pangola (Digitaria decumbens). O solo é classificado como Nitossolo Vermelho eutrófico de textura argilosa (BRASIL, 1972; EMBRAPA, 2006).

O solo foi destorroado manualmente e passado em peneira de $2,00 \mathrm{~mm}$ de abertura de malha e posteriormente separado nas classes de tamanho de agregados de 2,00-1,00; 1,00-0,50 e 0,50-0,25mm de diâmetro, com o auxílio de peneiras de abertura de malha específica (1,00, 0,50 e 0,25mm). Amostras das classes de agregados de solo foram submetidas a uma caracterização física inicial antes da implantação dos tratamentos, cujos resultados são apresentados na tabela 1. Esses resultados foram utilizados como valores de referência para as avaliações realizadas aos 90, 180, 270 e 360 dias após o plantio da gramínea.

O delineamento utilizado foi o inteiramente casualizado, em esquema fatorial $3 \times 2 \times 2 \times 4$, correspondendo a três classes de diâmetro de agregados (2,00-1,00; 1,00-0,50 e 0,50-0,25mm), duas condições de cultivo (com e sem gramínea), dois conteúdos de umidade (100\% e $60 \%$ de água disponível) e quatro épocas de avaliação (90, 180, 270 e 360 dias após o plantio da gramínea), com quatro repetições. Cada unidade experimental se constituiu de um vaso de polietileno preto, com capacidade para $10 \mathrm{~kg}$, o qual foi preenchido com $8,0 \mathrm{~kg}$ de cada classe de agregado seco ao ar.

A gramínea utilizada foi a Brachiaria ruziziensis Germain et Evrard, plantada nos vasos através de estolões, as quais foram irrigadas a cada dois dias, por meio de pesagens. Foram aplicados os conteúdos de água, calculados para atender os $60 \% \mathrm{e}$ $100 \%$ de água disponível, conforme cálculos baseados nos conteúdos de água na capacidade de campo $(0,033 \mathrm{MPa})$ e no ponto de murcha permanente $(1,5 \mathrm{MPa})$ do solo utilizado.

As amostragens foram realizadas a cada três meses $(90,180,270$ e 360 dias) para análises de tamanho 
Tabela 1 - Índices de agregação e de estabilidade de agregados e granulometria em amostra coletada na camada de 0-20cm de um Nitossolo Vermelho, em área cultivada com capim pangola (Digitaria decumbens) degradada.

\begin{tabular}{|c|c|c|c|c|c|c|}
\hline Classes de agregados & DMPAs & DMPAu & IEA & Areia & Silte & Argila \\
\hline $\mathrm{mm}$ & \multicolumn{2}{|c|}{-------------mm------------ } & & \multicolumn{3}{|c|}{ 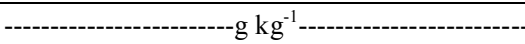 } \\
\hline $2,00-1,00$ & 1,471 & 0,495 & 0,336 & 358 & 193 & 449 \\
\hline $1,00-0,50$ & 0,712 & 0,316 & 0,444 & 383 & 197 & 420 \\
\hline $0,50-0,25$ & 0,351 & 0,203 & 0,578 & 377 & 195 & 428 \\
\hline
\end{tabular}

DMPAs - diâmetro médio ponderado de agregado seco; DMPAu - diâmetro médio ponderado de agregado úmido; IEA - estabilidade de agregado obtido pela relação diâmetro médio ponderado de agregado úmido e diâmetro médio ponderado de agregado seco.

e estabilidade de agregados. As amostras de solo retiradas dos vasos eram colocadas para secar à sombra e ao ar por 72 horas, sendo levemente destorroadas com as mãos. Em seguida, foram determinados: o diâmetro médio ponderado de agregados secos ao ar (DMPAs), conforme metodologia descrita em SILVA \& MIELNICZUK (1997); diâmetro médio ponderado de agregados estáveis em água (DMPAu), através do método descrito por TISDALL et al. (1978), adaptado por CARPENEDO \& MIELNICZUK (1990); e o índice de estabilidade dos agregados em água (IEA), obtido pela relação DMPAu/DMPAs conforme SILVA \& MIELNICZUK (1997).Os dados obtidos foram submetidos à análise de variância e, verificando-se efeito dos tratamentos pelo teste $\mathrm{F}$, as médias foram comparadas pelo teste de Tukey a $5 \%$ de probabilidade.

\section{RESULTADOS E DISCUSSÃO}

Os resultados da caracterização física inicial do Nitossolo Vermelho são apresentados na tabela 1, os quais foram utilizados como valores de referência para comparação com os resultados obtidos nas demais épocas de coleta como forma de avaliar o efeito dos tratamentos aplicados na formação e na estabilidade dos agregados.

Os valores de diâmetro médio ponderado de agregados secos ao ar (DMPAs) para a classe de diâmetro de agregados entre $0,50-0,25 \mathrm{~mm}$ foram elevados quando comparados com o valor inicial, de $0,351 \mathrm{~mm}$ (Tabela 1). No tratamento com gramínea, ocorreram em média acréscimos de 185 e $153 \%$, respectivamente, para 100 e $60 \%$ de água disponível, enquanto que, para o tratamento sem gramínea, os acréscimos foram de, respectivamente, 139 e $115 \%$. Neste caso, observa-se que a gramínea colaborou no processo de união de agregados menores para a formação de agregados maiores.

A classe de agregados com diâmetro entre 1,00-0,50mm também apresentou valores elevados em comparação com o seu valor inicial de caracterização $(0,712 \mathrm{~mm})$, em ambas as condições de cultivo (Tabela 2), principalmente no tratamento com $60 \%$ de água disponível. Nessa condição de umidade, ocorreram aumentos percentuais de 92 e $88 \%$, respectivamente, para o tratamento com e sem gramínea, superando o tratamento com $100 \%$ de água disponível, cujos aumentos foram, em média, de 83 e $79 \%$. O inverso ocorreu com a classe de agregados com diâmetro entre 2,00-1,00mm, em que os melhores resultados foram obtidos para $100 \%$ de água disponível, com percentuais médios de 50 e $35 \%$ para presença e ausência da gramínea, respectivamente. SALTON et al. (2008), também trabalhando com pastagens, verificaram que a massa de solo organizada em macroagregados maiores que $2,00 \mathrm{~mm}$ atingiu valores superiores a $55 \%$.

Na avaliação do diâmetro médio ponderado de agregado úmido (DMPAu) por classe de diâmetro de agregados, observou-se que a classe de agregados com diâmetro entre $0,50-0,25 \mathrm{~mm}$ apresentou aumento significativo nos seus valores (Tabela 3 ), principalmente para o tratamento com gramínea em que se aplicou $60 \%$ de água disponível, com acréscimo de 53\%. SILVA \& MIELNICZUK (1997), analisando a estabilidade de agregados em dois solos de textura argilosa com cultivo de gramíneas perenes (pangola e setária), verificaram que as raízes das gramíneas foram responsáveis pela união de agregados pequenos e formação de agregados maiores, por apresentarem maior densidade de raízes e melhor distribuição do sistema radicular no solo, promovendo a aproximação entre as partículas.

SALTON et al. (2008), trabalhando com diferentes sistemas de manejo e pastagens permanentes, observaram a distribuição da massa do solo em sete classes de tamanho de agregados estáveis em água e verificaram que os sistemas com presença de pastagem apresentaram, significativamente, maior quantidade de solo na camada de 0 a $5 \mathrm{~cm}$, constituindo agregados grandes (classe $>4,76 \mathrm{~mm}$ ), em relação aos demais sistemas, indicando o efeito do sistema radicular 
Tabela 2 - Diâmetro médio ponderado de três classes de diâmetro de agregados, determinado por peneiramento da amostra seca ao ar (DMPAs) nas combinações de duas condições de umidade, quatro épocas de amostragem, com e sem cultivo de braquiária.

\begin{tabular}{|c|c|c|c|c|c|}
\hline \multirow[t]{2}{*}{ Classe de agregados } & \multirow[t]{2}{*}{ Épocas de coleta } & \multicolumn{2}{|c|}{ 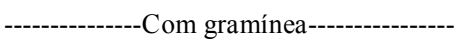 } & \multicolumn{2}{|c|}{ 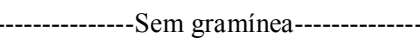 } \\
\hline & & $100 \% \mathrm{AD}$ & $60 \% \mathrm{AD}$ & $100 \% \mathrm{AD}$ & $60 \% \mathrm{AD}^{*}$ \\
\hline $\mathrm{mm}$ & dias & 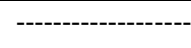 & 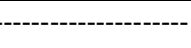 & ------------- & 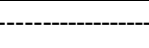 \\
\hline \multirow{4}{*}{$2,00-1,00$} & 90 & $2,312 \mathrm{aA} \alpha$ & $1,681 \mathrm{aB} \alpha$ & $1,854 \mathrm{aA} \alpha$ & $1,569 \mathrm{aA} \alpha$ \\
\hline & 180 & $2,339 \mathrm{aA} \alpha$ & $1,925 \mathrm{aA} \alpha$ & $1,922 \mathrm{aA} \alpha$ & $1,393 \mathrm{abA} \alpha$ \\
\hline & 270 & $2,254 \mathrm{aA} \alpha$ & $1,721 \mathrm{aA} \alpha$ & $2,235 \mathrm{aA} \alpha$ & $1,506 \mathrm{aB} \alpha$ \\
\hline & 360 & $1,926 \mathrm{aA} \alpha$ & $1,800 \mathrm{aA} \alpha$ & $1,914 \mathrm{aA} \alpha$ & $1,970 \mathrm{aA} \alpha$ \\
\hline \multirow{4}{*}{$1,00-0,50$} & 90 & $1,239 \mathrm{aA} \alpha$ & $1,342 \mathrm{aA} \alpha$ & $1,226 \mathrm{aA} \alpha$ & $1,280 \mathrm{aA} \alpha$ \\
\hline & 180 & $1,190 \mathrm{abA} \alpha$ & $1,422 \mathrm{aB} \alpha$ & $1,281 \mathrm{aA} \alpha$ & $1,378 \mathrm{aA} \alpha$ \\
\hline & 270 & $1,417 \mathrm{aA} \alpha$ & $1,390 \mathrm{aA} \alpha$ & $1,256 \mathrm{aA} \alpha$ & $1,363 \mathrm{aA} \alpha$ \\
\hline & 360 & $1,369 \mathrm{aA} \alpha$ & $1,308 \mathrm{aA} \alpha$ & $1,335 \mathrm{aA} \alpha$ & $1,319 \mathrm{aA} \alpha$ \\
\hline \multirow{4}{*}{$0,50-0,25$} & 90 & $0,930 \mathrm{aA} \alpha$ & $0,810 \mathrm{aA} \alpha$ & $0,805 \mathrm{aA} \alpha$ & $0,813 \mathrm{aA} \alpha$ \\
\hline & 180 & $0,980 \mathrm{aA} \alpha$ & $0,902 \mathrm{aA} \alpha$ & $0,792 \mathrm{aA} \alpha$ & $0,716 \mathrm{aA} \alpha$ \\
\hline & 270 & $0,993 \mathrm{aA} \alpha$ & $0,965 \mathrm{aA} \alpha$ & $0,900 \mathrm{aA} \alpha$ & 0,757 aA $\beta$ \\
\hline & 360 & $1,110 \mathrm{aA} \alpha$ & $0,880 \mathrm{aB} \alpha$ & $0,861 \mathrm{aA} \beta$ & $0,728 \mathrm{aA} \alpha$ \\
\hline
\end{tabular}

*AD - água disponível

Médias seguidas pela mesma letra minúscula na linha, maiúscula entre condições de umidade dentro de cada tratamento com e sem braquiária e grega na coluna dentro de cada classe de agregados não diferem a $5 \%$ de probabilidade pelo teste de Tukey.

da gramínea no processo de formação dos macroagregados.

As classes de agregados com diâmetro entre 2,00-1,00 e 1,00-0,50mm também apresentaram maiores valores médios de DMPAu para o tratamento com gramínea, sendo que a classe de agregados com diâmetro entre $1,00-0,50 \mathrm{~mm}$ não diferiu significativamente entre os conteúdos de umidade 100 e $60 \%$, com acréscimos de $46 \%$ para ambos. Para o tratamento sem gramínea, a classe de agregados com diâmetro entre 2,00-1,00mm apresentou redução em seus valores em comparação com seu valor inicial de caracterização, de $0,495 \mathrm{~mm}$ (Tabela 1), com decréscimos percentuais de $9 \%$ para 100 e $60 \%$ de água disponível, indicando elevada vulnerabilidade dos agregados formados, detectado através da separação por peneiramento da amostra seca (Tabela 2).

Com relação à agregação do solo, medida por peneiramento da amostra em água, verifica-se que, apesar dos valores baixos, houve aumento no tratamento com gramínea, confirmando o efeito significativo do sistema radicular da braquiária na formação e estabilização de agregados, demonstrada tanto pelos valores obtidos por peneiramento da amostra seca ao ar (Tabela 2) e em água (Tabela 3 ). PALADINI \& MIELNICZUK (1991) e SILVA \&
MIELNICZUK (1998) também observaram a ação eficiente de uma gramínea tropical perene, o capim pangola (Digitaria decumbens), sobre a agregação do solo.

Na tabela 4, são apresentados os valores do índice de estabilidade dos agregados, calculados através da relação DMPAu/DMPAs. Verifica-se que a classe de agregados com diâmetro entre $0,50-0,25 \mathrm{~mm}$ apresentou aumento significativo do diâmetro médio para todos os tratamentos, quando comparados com o valor inicial de caracterização, de 0,578mm (Tabela 1), demonstrando maior estabilidade dos agregados. A classe de agregados com diâmetro entre $1,00-0,50 \mathrm{~mm}$ apresentou valores superiores à caracterização inicial, de $0,444 \mathrm{~mm}$ (Tabela 1), principalmente para o tratamento com gramínea, com aumento percentual de $46 \%$ para as duas condições de água disponível. VEZZANI \& MIELNICZUK (2011), trabalhando em ecossistema nativo composto por várias espécies de gramíneas, verificaram que o solo estudado apresentou em torno de $76,4 \%$ de solo agregado, sendo $63,8 \%$ desse total composto por macroagregados maiores que $0,25 \mathrm{~mm}$, fato atribuído à longa e contínua atuação do sistema radicular das gramíneas.

Para o tratamento sem gramínea, os agregados com diâmetro entre 2,00-1,00mm

Ciência Rural, v.42, n.7, jul, 2012. 
Tabela 3 - Diâmetro médio ponderado de três classes de diâmetro de agregados, obtidos por peneiramento via úmida (DMPAu) nas combinações de duas condições de umidade, quatro épocas de amostragem, com e sem cultivo de braquiária.

\begin{tabular}{|c|c|c|c|c|c|}
\hline \multirow[t]{2}{*}{ Classe de agregados } & \multirow[t]{2}{*}{ Épocas de coleta } & \multicolumn{2}{|c|}{------------Com gramínea--------------- } & \multicolumn{2}{|c|}{--------------Sem gramínea------------- } \\
\hline & & $100 \% \mathrm{AD}$ & $60 \% \mathrm{AD}$ & $100 \% \mathrm{AD}$ & $60 \% \mathrm{AD}^{*}$ \\
\hline $\mathrm{mm}$ & dias & \multicolumn{4}{|c|}{ 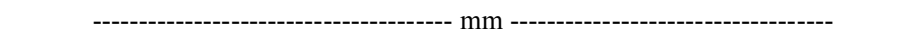 } \\
\hline \multirow{4}{*}{$2,00-1,00$} & 90 & $0,570 \mathrm{aA} \alpha$ & $0,587 \mathrm{aA} \alpha$ & $0,499 \mathrm{aA} \alpha$ & $0,518 \mathrm{aA} \alpha$ \\
\hline & 180 & $0,579 \mathrm{aA} \alpha$ & $0,529 \mathrm{aA} \alpha$ & $0,417 \mathrm{aA} \beta$ & $0,466 \mathrm{aA} \alpha$ \\
\hline & 270 & $0,627 \mathrm{aA} \alpha$ & $0,598 \mathrm{aA} \alpha$ & $0,446 \mathrm{aA} \beta$ & $0,410 \mathrm{abA} \beta$ \\
\hline & 360 & $0,567 \mathrm{aA} \alpha$ & $0,560 \mathrm{aA} \alpha$ & 0,437 aA $\beta$ & $0,407 \mathrm{abA} \beta$ \\
\hline \multirow{4}{*}{$1,00-0,50$} & 90 & $0,483 \mathrm{aA} \alpha$ & $0,515 \mathrm{aA} \alpha$ & $0,370 \mathrm{aB} \beta$ & $0,505 \mathrm{aA} \alpha$ \\
\hline & 180 & $0,391 \mathrm{bA} \alpha$ & $0,454 \mathrm{aA} \alpha$ & $0,309 \mathrm{aB} \alpha$ & $0,415 \mathrm{bA} \alpha$ \\
\hline & 270 & $0,518 \mathrm{aA} \alpha$ & $0,457 \mathrm{aA} \alpha$ & $0,298 \mathrm{aB} \beta$ & $0,415 \mathrm{bA} \alpha$ \\
\hline & 360 & $0,453 \mathrm{abA} \alpha$ & $0,414 \mathrm{abA} \alpha$ & $0,315 \mathrm{aA} \beta$ & $0,349 \mathrm{bA} \alpha$ \\
\hline \multirow{4}{*}{$0,50-0,25$} & 90 & $0,320 \mathrm{aA} \alpha$ & $0,345 \mathrm{aA} \alpha$ & $0,316 \mathrm{aA} \alpha$ & $0,289 \mathrm{aA} \beta$ \\
\hline & 180 & $0,240 \mathrm{bA} \alpha$ & $0,282 \mathrm{bA} \alpha$ & $0,277 \mathrm{aA} \alpha$ & $0,243 \mathrm{abA \alpha}$ \\
\hline & 270 & $0,309 \mathrm{aA} \alpha$ & $0,303 \mathrm{abA} \alpha$ & $0,280 \mathrm{aA} \alpha$ & $0,234 \mathrm{bA} \beta$ \\
\hline & 360 & $0,293 \mathrm{aA} \alpha$ & $0,347 \mathrm{aB} \alpha$ & $0,247 \mathrm{abA} \alpha$ & $0,219 \mathrm{bA} \beta$ \\
\hline
\end{tabular}

*AD - água disponível

Médias seguidas pela mesma letra minúscula na linha, maiúscula entre condições de umidade dentro de cada tratamento com e sem braquiária e grega na coluna dentro de cada classe de agregados não diferem a $5 \%$ de probabilidade pelo teste de Tukey.

Tabela 4 - Índice de estabilidade de agregados em três classes de diâmetro de agregados nas combinações de duas condições de umidade, quatro épocas de amostragem, com e sem cultivo de braquiária.

\begin{tabular}{|c|c|c|c|c|c|}
\hline \multirow[t]{2}{*}{ Classes de agregados } & \multirow[t]{2}{*}{ Épocas de coleta } & \multicolumn{2}{|c|}{----------Com gramínea-------------- } & \multicolumn{2}{|c|}{-------------Sem gramínea------------- } \\
\hline & & $100 \% \mathrm{AD}$ & $60 \% \mathrm{AD}$ & $100 \% \mathrm{AD}$ & $60 \% \mathrm{AD}^{*}$ \\
\hline $\mathrm{mm}$ & dias & & & & \\
\hline \multirow{4}{*}{$2,00-1,00$} & 90 & $0,387 \mathrm{aA}$ & $0,399 \mathrm{aA}$ & $0,339 \mathrm{aA}$ & $0,352 \mathrm{aA}$ \\
\hline & 180 & $0,394 \mathrm{aA}$ & $0,359 \mathrm{aA}$ & $0,283 \mathrm{aB}$ & $0,317 \mathrm{abA}$ \\
\hline & 270 & $0,427 \mathrm{aA}$ & $0,406 \mathrm{aA}$ & $0,304 \mathrm{aB}$ & $0,279 \mathrm{bB}$ \\
\hline & 360 & $0,386 \mathrm{aA}$ & $0,380 \mathrm{aA}$ & $0,297 \mathrm{aB}$ & $0,277 \mathrm{bB}$ \\
\hline \multirow{4}{*}{$1,00-0,50$} & 90 & $0,679 \mathrm{aA} \alpha$ & $0,724 \mathrm{aA} \alpha$ & $0,519 \mathrm{aA} \beta$ & $0,709 \mathrm{aA} \alpha$ \\
\hline & 180 & $0,549 \mathrm{bA} \alpha$ & $0,638 \mathrm{aA} \alpha$ & $0,434 \mathrm{aA} \alpha$ & $0,583 \mathrm{bA} \alpha$ \\
\hline & 270 & $0,728 \mathrm{abA} \alpha$ & $0,641 \mathrm{aA} \alpha$ & $0,419 \mathrm{aB} \beta$ & $0,583 \mathrm{bA} \alpha$ \\
\hline & 360 & $0,636 \mathrm{aA} \alpha$ & $0,581 \mathrm{abA} \alpha$ & $0,442 \mathrm{aB} \beta$ & $0,490 \mathrm{bA} \alpha$ \\
\hline \multirow{4}{*}{$0,50-0,25$} & 90 & $0,911 \mathrm{aA} \alpha$ & $0,983 \mathrm{aA} \alpha$ & $0,899 \mathrm{aA} \alpha$ & $0,824 \mathrm{aA} \alpha$ \\
\hline & 180 & $0,684 \mathrm{bA} \alpha$ & $0,803 \mathrm{abA} \alpha$ & $0,791 \mathrm{aA} \alpha$ & 0,692 aA $\beta$ \\
\hline & 270 & $0,880 \mathrm{abA} \alpha$ & $0,864 \mathrm{aA} \alpha$ & $0,797 \mathrm{aA} \alpha$ & $0,667 \mathrm{abA} \beta$ \\
\hline & 360 & $0,834 \mathrm{abA} \alpha$ & $0,990 \mathrm{abB} \alpha$ & $0,703 \mathrm{abA} \alpha$ & $0,625 \mathrm{abA} \beta$ \\
\hline
\end{tabular}

*AD - água disponível.

Médias seguidas pela mesma letra minúscula na linha, maiúscula entre condições de umidade dentro de cada tratamento com e sem braquiária e grega na coluna dentro de cada classe de agregados não diferem a $5 \%$ de probabilidade pelo teste de Tukey.

Ciência Rural, v.42, n.7, jul, 2012. 
apresentaram valores médios da relação DMPAu/ DMPAs inferiores ao valor da caracterização inicial nas duas condições de água disponível. As classes de agregados com diâmetro entre 1,00-0,50 e 0,50-0,25mm também apresentaram valores baixos para o tratamento sem gramínea, porém com aumento percentual positivo, sendo que a classe de agregados de 1,00-0,50mm apresentou aumento de $33 \%$ com $60 \%$ de água disponível, enquanto a classe de agregados entre 0,50 $0,25 \mathrm{~mm}$ apresentou aumento de $38 \%$ com $100 \%$ de água disponível.

Esse efeito deve-se, provavelmente, aos ciclos sucessivos de umedecimento e secagem do solo para o tratamento sem gramínea, que podem ter contribuído na estabilidade de agregados. Segundo HORN \& DEXTER (1988); HORN (1990), esses ciclos levam à desidratação de ligantes orgânicos e inorgânicos do solo, que proporcionam a formação de agregados de maior tamanho. OLIVEIRA et al. (1996) trabalharam com ciclos de umedecimento e secagem em latossolos brasileiros e concluíram que o efeito desses, sobre os agregados maiores, na camada superficial do solo, são maiores.

\section{CONCLUSÃO}

Os maiores valores de diâmetro médio ponderado de agregado úmido e do índice de estabilidade de agregados para o tratamento com gramínea indicam que o sistema radicular da Brachiaria ruziziensis Germain et Evrard favoreceu a formação e estabilização dos agregados de solo com maiores incrementos nas classes de agregados de menor diâmetro, decrescendo os efeitos com aumento do tamanho dos agregados. O maior conteúdo de água disponível no solo contribuiu para maiores valores de diâmetro médio ponderado de agregado úmido e do índice de estabilidade de agregados na presença da gramínea.

\section{REFERÊNCIAS}

BOCHNER, J.K. et al. Matéria orgânica e agregação de um planossolo sob diferentes coberturas florestais. Cerne, v.14, n.1, p.46-53, 2008.

BRASIL. Ministério da Agricultura. Levantamento explolatório - reconhecimento de solos do Estado da Paraíba. Rio de Janeiro: MA/SUDENE, 1972. 683p.

CAMARGO FILHO, S.T. Recuperação de áreas degradadas no município de Paty do Alferes pela introdução de gramíneas forrageiras e leguminosas arbóreas. 1999. 105f. Dissertação (Mestrado em Zootecnia) - UFRRJ, Seropédica, RJ.
CARPENEDO, V.; MIELNICZUK, J. Estado de agregação e qualidade de agregados de Latossolos roxos, submetidos a diferentes sistemas de manejo. Revista Brasileira de Ciência do Solo, v.14, n.1, p.99-105, 1990.

CASTRO FILHO, C. et al. Estabilidade dos agregados e sua relação com o teor de carbono orgânico em um Latossolo Roxo distrófico, em função de sistemas de plantio, rotações de culturas e métodos de preparo das amostras. Revista Brasileira de Ciência do Solo, v.22, n.3, p.527- 538, 1998.

EDWARDS, A.P.; BREMNER, J.M. Microaggregates in soils. Journal Soil Science, v.18, p.64-73, 1967. Disponível em: $<$ http://onlinelibrary.wiley.com/doi/10.1111/j.13652389.1967.tb01488.x>. Acesso em: 15 fev. 2012. doi: 10.1111/j.1365-2389.1967.tb01488.x.

EMBRAPA. Centro Nacional de Pesquisa de Solos. Sistema brasileiro de classificação de solos. 2.ed. Rio de Janeiro, 2006. 306p.

HORN, R. Aggregate characterization as compared to soil bulk properties. Soil and Tillage Research, v.17, p.265-289, 1990. Disponível em: <http://www.sciencedirect.com/science/ article/pii/016719879090041B >. Acesso em: $27 \mathrm{dez} .2011$. doi: 10.1016/0167-1987(90)90041-B.

HORN, R.; DEXTER, R.A. Dynamics of soil aggregation in an irrigated desert loess. Soil and Tillage Research, v.13, p.253266, 1988. Disponível em: <http://www.sciencedirect.com/ science/article/pii/0167198789900020>. Acesso em: $13 \mathrm{fev}$. 2012. doi: 10.1016/0167-1987(89)90002-0.

MIELNICZUK, J. Importância do estudo de raízes no desenvolvimento de sistemas agrícolas sustentáveis. In: WORKSHOP SOBRE SISTEMA RADICULAR: METODOLOGIAS E ESTUDO DE CASO, 1999, Aracaju, SE. Anais... Aracaju: EMBRAPA Tabuleiros Costeiros, 1999. p.13-17.

OLIVEIRA, T.S et al. Efeito de ciclos de umedecimento e secagem sobre a estabilidade de agregados em água de quatro latossolos brasileiros. Revista Brasileira de Ciência do Solo, v.20, p.509-515, 1996.

PALADINI, F.L.S.; MIELNICZUK, J. Distribuição de tamanho de agregados de um podzólico vermelho-escuro afetado por sistemas de cultivos. Revista Brasileira de Ciência do Solo, v.15, n.2, p.135-140, 1991.

PALMEIRA, P.R.T. et al. Agregação de um Planossolo submetido a diferentes sistemas de cultivo. Revista Brasileira de Ciência do Solo, v.23, n.2, p.189-195, 1999.

SALTON, J.C. et al. Agregação e estabilidade de agregados do solo em sistemas agropecuários em Mato Grosso do Sul. Revista Brasileira de Ciência do solo, v.32, n.1, p.11-21, 2008. Disponível em: $<$ http://www.scielo.br/scielo.php?script=sci_pdf\&pid=S0100$06832008000100002 \& \operatorname{lng}=\mathrm{pt} \& \mathrm{nrm}=\mathrm{iso} \& \operatorname{tng}=\mathrm{pt}>$. Acesso em: 21 jul 2011. doi: 10.1590/S0100-06832008000100001.

SILVA, I.F.; MIELNICZUK, J. Sistemas de cultivo e características do solo afetando a estabilidade de agregados. Revista Brasileira de Ciência do Solo, v.22, p.311-317, 1998.

SILVA, I.F.; MIELNICZUK, J. Ação do sistema radicular de plantas na formação e estabilização de agregados. Revista 
Brasileira de Ciência do Solo, v.21, n.2, p.113-117, 1997. Disponível em: <http://www.scielo.br/scielo.php?pid=S0100$06832004000500011 \&$ script $=$ sci_abstract $\&$ tlng $=\mathrm{pt}>$. Acesso em: 21 jul. 2011. doi: 10.1590/S0100-06832008000100002.

SILVA, M.L.N. et al. Estabilidade e resistência de agregados de um Latossolo Vermelho-Escuro cultivado com sucessão milhoadubo verde. Pesquisa Agropecuária Brasileira, v.33, n.1, p.97-103, 1998.

TISDALL, J.M. Possible role of soil microorganisms in aggregation in soils. Plant Soil, v.159, p.115-121, 1994. Disponível em: <http://www.springerlink.com/content/ 18589wm47561w713>. Acesso em: 14 fev. 2012. doi: 10.1007/ BF00000100.

TISDALL, J.M.; OADES, J.M. Organic matter and water-stable aggregates in soils. Journal of Soil Science, v.33, p.141-163, 1982. Disponível em: <http://onlinelibrary.wiley.com/doi/ 10.1111/j.1365-2389.1982.tb01755.x>. Acesso em: $27 \mathrm{dez}$ 2011. doi: $10.1111 / \mathrm{j} .1365-2389.1982 . t b 01755$.x.
TISDALL, J.M. et al. The stability of soil aggregates as affected by organic materials microbial activity and physical disruption. Australian Journal of Soil Research, v.16, p.9-17, 1978. Disponível em: <http://www.publish.csiro.au/paper/SR9780009.htm>. Acesso em: 27 dez. 2011. doi:10.1071/SR9780009.

VEZZANI, F.M.; MIELNICZUK, J. Agregação e estoque de carbono em argissolo submetido a diferentes práticas de manejo agrícola. Revista Brasileira de Ciência do Solo, v.35, n.1, p.213-223, 2011. Disponível em: <http://www.scielo.br/ scielo.php? script=sci_pdf\&pid=S 010006832 $011000100020 \& \operatorname{lng}=\mathrm{pt} \& \mathrm{nrm}=\overline{\mathrm{i}} \mathbf{p} \& \mathrm{t} \operatorname{lng}=\mathrm{pt}>$. Acesso em: 18 ago. 2011. doi: 10.1590/S0100-06832011000100020.

WOHLENBERG, E.V. et al. Dinâmica da agregação de um solo franco-arenoso em cinco sistemas de culturas em rotação em rotação e em sucessão. Revista Brasileira de Ciência do Solo, v.28, n.5, p.891-900, 2004. Disponível em: <http:// www.scielo.br/scielo.php?pid=S 01000683200400 $0500011 \&$ script $=$ sci_abstract\&tlng=pt $>$. Acesso em: $21 \mathrm{jul}$ 2011. doi:10.1590/S0100-06832004000500011. 